\section{P1-293 INVOLVING LOCAL COMMUNITY: TESTING MODELS FOR COMMUNICATING SURVEILLANCE DATA. FROM PLANNING TO ELABORATING AND EVALUATING EFFECTIVE COMMUNICATIVE TOOLS TO SPECIFIC TARGET GROUPS AT LOCAL LEVEL}

doi:10.1136/jech.2011.142976e.85

\begin{abstract}
${ }^{1} \mathrm{~V}$ Possenti, ${ }^{*}{ }^{1} \mathrm{~B}$ D Mei, ${ }^{1} \mathrm{C}$ Cattaneo, ${ }^{1} \mathrm{I}$ Giovannelli, ${ }^{1} \mathrm{P}$ D'Argenio, ${ }^{2} \mathrm{E}$ Benelli, ${ }^{2} \mathrm{~S}$ Menna, ${ }^{1} \mathrm{~S}$ Salmaso, ${ }^{1} \mathrm{G}$ Ferrante, ${ }^{1} \mathrm{~V}$ Minardi, 'E Quarchioni, ${ }^{1} \mathrm{~S}$ Baldissera, ${ }^{3} \mathrm{~N}$ Bertozzi, ${ }^{4} \mathrm{G}$ Carrozzi, ${ }^{5} \mathrm{~A}$ D'Argenzio, ${ }^{6} \mathrm{P}$ Fateh-Moghadam, ${ }^{7} \mathrm{M} 0$ Trinito, ${ }^{8} \mathrm{~S}$ Vasselli, ${ }^{9} \mathrm{~S}$ Campostrini. ${ }^{1}$ Istituto Superiore di Sanità, Rome, Italy; ${ }^{2}$ Zadig-Rome, Rome, Italy; ${ }^{3}$ LHU Cesena, Cesena, Italy; ${ }^{4}$ LHU Modena, Modena, Italy; ${ }^{5}$ LHU Caserta, Caserta, Italy; ${ }^{6}$ LHU Trento, Trento, Italy; ${ }^{7}$ LHU Rome C, Rome, Italy; ${ }^{8}$ Ministry of Health, Rome, Italy; ${ }^{9}$ University of Venice, Venice, Italy
\end{abstract}

Introduction When monitoring population health-related behaviours it is important to assess the effectiveness of delivering health related information to specific target groups such that any need for improved communication can be identified. The Italian Behavioural Risk Factors Surveillance System Passi is conducted through Local Health Units (LHUs) personnel interviewing a random sample of 18-69 year olds monthly. PinC, the Programme of Information and Communication for Gaining Health, promoted a training project for Passi care workers the aim of which was to achieve competency in communication using methods that were pre-tested and effective.

Methods Forty operators from 25 LHUs participated in the sixmonthly-training: three one day meetings (22 April 2010, 24 June 2010, 11 November 2010) and two rounds of two-monthly-activities in the field. A password-protected portal was used for exchanging materials among participants at a distance.

Results The seven inter-regional Working Groups planned communication, performed and tested materials using surveillance results for specific target groups at a local level. The need for effective approaches to communication was highlighted. Draft materials (leaflets for older women, posters for young people, charts for GPs, papers for policy makers/stakeholders) were tested on selected target groups. The two lay target populations (women, children) evaluated the tools positively, critiquing images, recommending clear and essential messages, clarifying what should be done to improve health. The professional groups suggested editing of text and space for graphs/tables. Materials will be modified accordingly and delivered.

Conclusion Surveillance systems are valuable tools for advocacy at a local level. Communication is fundamental for knowledge, awareness and empowerment processes in specific target groups, professional and lay. To be effective, materials must be carefully planned and tested.

\section{P1-294 RISK FACTORS FOR POOR MENTAL HEALTH DURING PREGNANCY FROM THE BORN IN BRADFORD COHORT: EARLY RESULTS FROM A BI-ETHNIC SAMPLE}

doi:10.1136/jech.2011.142976e.86

\begin{abstract}
${ }^{1} \mathrm{~S}$ Prady, ${ }^{*}{ }^{2} \mathrm{~L}$ Fairley, ${ }^{1} \mathrm{~K}$ Bloor, ${ }^{1} \mathrm{~S}$ Gilbody, ${ }^{1} \mathrm{~K}$ Kiernan, ${ }^{1} \mathrm{~K}$ Pickett, ${ }^{2} \mathrm{P}$ Raynor, ${ }^{2} \mathrm{~J}$ Wright. ${ }^{1}$ University of York, York, UK; ${ }^{2}$ Bradford Institute for Health Research, Bradford, UK
\end{abstract}

Introduction Born in Bradford is a large multi-ethnic birth cohort drawn from the North of England. We describe the risk factors associated with poor mental health in pregnancy for White and Pakistani-origin women.
Methods We examined data from White $(\mathrm{N}=1901)$ and Pakistani $(\mathrm{N}=1360)$ women who completed the GHO-28 in English. We used univariate and multivariate logistic regression to examine sociodemographic risk factors associated with scoring above the conventional threshold for poor mental health. We conducted a sensitivity analysis on the threshold method by comparing the highest and lowest scoring quintile within each ethnic group.

Results $43 \%$ of White and $47 \%$ of Pakistani women scored above the threshold. For White women univariate risk factors were not living with a partner, self-reported financial worries and less education. For Pakistani women not being in a relationship and having financial worries were associated with poor mental health. Significant variables remaining in the multivariate models for both groups were financial worries (White OR 2.4, 95\% CI 2.0 to 3.0; Pakistani 2.7, 2.1 to 3.5 ) and not being in a relationship (White $1.4,1.0$ to 2.1; Pakistani 2.6, 1.0 to 6.5). Sensitivity analyses indicated the same significant risk factors with the addition of cohabitation and younger age in the univariate analyses for White women. Pakistani women had worse within-quintile scores than White women (highest quintile difference 5.6 points, $t=8.8$, $\mathrm{p}<0.0001$ ).

Conclusion Prevalence and severity of poor mental health varies by ethnicity but consistency in the associated risk factors underline patterns of social disadvantage in both groups.

\section{P1-295 SCREENING FOR INFLUENZA AT THE BORDER: IS IT WORTHWHILE?}

doi:10.1136/jech.2011.142976e.87

${ }^{1} \mathrm{P}$ Priest, ${ }^{*} \mathrm{~L} \mathrm{~L}$ Jennings, ${ }^{3} \mathrm{~A}$ Duncan, ${ }^{4} \mathrm{C}$ Brunton, ${ }^{5} \mathrm{M}$ Baker. ${ }^{1}$ University of Otago, Dunedin, New Zealand; ${ }^{2}$ Canterbury Health Laboratories, Christchurch, New Zealand; ${ }^{3}$ Canterbury District Health Board, Christchurch, New Zealand; ${ }^{4}$ University of Otago, Christchurch, New Zealand; ${ }^{5}$ University of Otago, Wellington, New Zealand

Introduction The aim of border screening for influenza is to prevent or delay the entry of a new pandemic strain, but the evidence base for this strategy is limited. This study aimed to assess the test characteristics of a health questionnaire and temperature measurement for predicting influenza infection in arriving international travellers.

Methods A questionnaire was distributed on board flights from Australia to Christchurch, New Zealand during 12 weeks in June-September 2008. It included questions on demographics, symptoms, contacts, and countries visited. All symptomatic travellers and a random sample of asymptomatic travellers were asked to provide a throat swab and have their temperature measured.

Results 175/307 (57\%) flights were screened according to protocol, and 15618/22 $192(70 \%)$ travellers on screened flights returned questionnaires. $48 \%$ of symptomatic travellers and $35 \%$ of sampled asymptomatic travellers agreed to provide a swab. The overall prevalence of influenza infection was $1 \%$. The sensitivity for influenza infection of 'any symptom' was $85 \%$, for cough $58 \%$, sore throat $26 \%$, self reported fever $15 \%$, and for measured temperature $>37.8^{\circ} \mathrm{C}$ it was $8 \%$. The highest PPV was for self-reported fever, at $23 \%$.

Conclusion The poor sensitivity of most screening questions or tests and low prevalence of influenza infection among travellers means that border screening would be resource-intensive yet would fail to identify all, or possibly even most, influenza infected travellers entering the country. 